\title{
Indecomposable decomposition and couniserial dimension
}

\author{
A. Ghorbani · S. K. Jain · Z. Nazemian
}

Received: 8 October 2014 / Revised: 8 November 2014 / Accepted: 14 November 2014 /

Published online: 27 November 2014

(C) The Author(s) 2014. This article is published with open access at SpringerLink.com

\begin{abstract}
Dimensions like Gelfand, Krull, Goldie have an intrinsic role in the study of theory of rings and modules. They provide useful technical tools for studying their structure. We define and study couniserial dimension for modules. Couniserial dimension is a measure of how far a module deviates from being uniform. Despite their different objectives, it turns out that there are certain common properties between the couniserial dimension and Krull dimension. Among others, each module having such a dimension contains a uniform submodule and has finite uniform dimension. Like all dimensions, this is an ordinal valued invariant. Every module of finite length has couniserial dimension and its value lies between the uniform dimension and the length of the module. Modules with countable couniserial dimension are shown to possess indecomposable decomposition. In particular, a von Neumann regular ring with countable couniserial dimension is semisimple artinian. If the maximal right
\end{abstract}

Communicated by Efim Zelmanov.

This paper was presented at the OSU-Denison conference, May 10, 2014 and at the University of California, San Diego, June 21, 2014.

A. Ghorbani $(\varangle) \cdot$ Z. Nazemian

Department of Mathematical Sciences, Isfahan University of Technology, P.O.Box: 84156-83111, Isfahan, Iran

e-mail: a_ghorbani@cc.iut.ac.ir

Z. Nazemian

e-mail: z.nazemian@math.iut.ac.ir

S. K. Jain

Department of Mathematics, Ohio University, Athens, OH 45701, USA

e-mail: jain@ohio.edu

S. K. Jain

King Abdulaziz University, Jeddah, Saudi Arabia 
quotient ring of a semiprime right non-singular ring $R$ has a couniserial dimension as an $R$-module, then $R$ is a semiprime right Goldie ring. As one of the applications, it follows that all right $R$-modules have couniserial dimension if and only if $R$ is a semisimple artinian ring.

Keywords Uniform module - Maximal right quotient ring - Indecomposable decomposition · Uniserial dimension - Couniserial dimension · Von Neumann regular ring $\cdot$ Semisimple module

Mathematics Subject Classification Primary 16D70 $\cdot 16 D 90 \cdot 16$ P70 ; Secondary $03 \mathrm{E} 10 \cdot 13 \mathrm{E} 10$

\section{Introduction}

In this article we introduce a notion of dimension of a module, to be called couniserial dimension. It is an ordinal valued invariant that is in some sense a measure of how far a module is from being uniform. In order to define couniserial dimension for modules over a ring $R$, we first define, by transfinite induction, classes $\zeta_{\alpha}$ of $R$-modules for all ordinals $\alpha \geq 1$. First we remark that if a module $M$ is isomorphic to all its non-zero submodules, then $M$ must be uniform. To start with, let $\zeta_{1}$ be the class of all uniform modules. Next, consider an ordinal $\alpha>1$; if $\zeta_{\beta}$ has been defined for all ordinals $\beta<\alpha$, let $\zeta_{\alpha}$ be the class of those $R$-modules $M$ such that for every non-zero submodule $N$ of $M$, where $N \supsetneqq M$, we have $N \in \bigcup_{\beta<\alpha} \zeta_{\beta}$. If an $R$-module $M$ belongs to some $\zeta_{\alpha}$, then the least such $\alpha$ is called the couniserial dimension of $M$, denoted by c.u.dim( $(M)$. For $M=0$, we define c.u.dim $(M)=0$. If a non-zero module $M$ does not belong to any $\zeta_{\alpha}$, then we say that c.u.dim $(M)$ is not defined, or that $M$ has no couniserial dimension. Equivalently, Proposition 3.3 shows that an $R$-module $M$ has couniserial dimension if and only if for each descending chain of submodules of $M$, $M_{1} \geq M_{2} \geq \cdots$, there exists $n \geq 1$, either $M_{n}$ is uniform or $M_{n} \cong M_{k}$ for all $k \geq n$. It is clear by the definition that every submodule and so every summand of a module with couniserial dimension has couniserial dimension. Also note that, for the integer number $n$, couniserial dimension of $\mathbb{Z}^{n}$ is $n$. An example is given to show that the direct sum of two modules each with couniserial dimension (even copies of a module) need not have couniserial dimension. In Sect. 3, we prove some basic properties of the couniserial dimension. In Sect. 4, we prove our main results. It is shown in Theorem 4.3 that a module of countable (finite or infinite) couniserial dimension can be decomposed into indecomposable modules. Theorem 4.5 shows that a Dedekind finite module with couniserial dimension is a finite direct sum of indecomposable modules. Theorem 4.10 in Sect. 4 shows that for a semiprime right non-singular ring $R$ with maximal right quotient ring $Q$, if $Q_{R}$ has couniserial dimension, then $R$ is a semiprime right Goldie ring which is a finite product of piecewise domains. The reader may compare this with the well known result that a prime ring with Krull dimension is a right Goldie ring but need not be a piecewise domain. Furthermore, a prime right Goldie ring need not have couniserial dimension as is also the case for Krull dimension.

In Sect. 5, we give some applications of couniserial dimension. It is shown in Proposition 5.2 that a module $M$ with finite length is semisimple if and only if for 
every submodule $N$ of $M$ the right $R$-module $\oplus_{i=1}^{\infty} M / N$ has couniserial dimension. As a consequence a commutative noetherian ring $R$ is semisimple if and only if for every finite length module $M$ the module $\oplus_{i=1}^{\infty} M$ has couniserial dimension. It is shown in Proposition 5.4 that if $P$ is an anti-coHopfian projective right $R$-module and $\oplus_{i=1}^{\infty} E(P)$ has couniserial dimension, then $P$ is injective. As another application we show that all right (left) $R$-module have couniserial dimension if and only if $R$ is semisimple artinian (see Theorem 5.8). Several examples are included in the paper that demonstrates as to why the conditions imposed are necessary.

\section{Definitions and notations}

Recall that a semisimple module $M$ is said to be homogeneous if $M$ is a direct sum of pairwise isomorphic simple submodules. A module $M$ has finite uniform dimension (or finite Goldie rank) if $M$ contains no infinite direct sum of non-zero submodules, or equivalently, there exist independent uniform submodules $U_{1}, \ldots, U_{n}$ in $M$ such that $\oplus_{i=1}^{n} U_{i}$ is an essential submodule of $M$. Note that $n$ is uniquely determined by $M$. In this case, it is written $\mathrm{u} \cdot \operatorname{dim}(M)=n$.

For any module $M$, we define $\mathrm{Z}(M)=\{x \in M: \operatorname{ann}(x)$ is an essential right ideal of $R\}$. It can be easily checked that $\mathrm{Z}(M)$ is a submodule of $M$. If $\mathrm{Z}(M)=0$, then $M$ is called a non-singular module. In particular, if we take $M=R_{R}$, then $R$ is called right non-singular if $\mathrm{Z}\left(R_{R}\right)=0$.

A ring $R$ is called a right Goldie ring if it satisfies the following two conditions: (i) $R$ has ascending chain condition on right annihilator ideals and, (ii) $\operatorname{u} \operatorname{dim}\left(R_{R}\right)$ is finite. Recall that a ring $R$ is right $V$-ring if all right simple $R$-modules are injective. A ring $R$ is called fully right idempotent if $I=I^{2}$, for every right ideal $I$. We recall that a right V-ring is fully right idempotent (see [19, Corollary 2.2]) and a prime fully right idempotent ring is right non-singular (see [2, Lemma 4.3]). So a prime right V-ring is right non-singular. Recall that a module $M$ is called $\Sigma$-injective if every direct sum of copies of $M$ is injective. A ring $R$ is called right $\Sigma$ - $V$-ring if each simple right module is $\Sigma$-injective.

In this paper, for a ring $R, Q=Q_{\max }(R)$ stands by the maximal right quotient ring $R$. It is well known that if $R$ is a right non-singular, then the injective hull of $R_{R}$, $E\left(R_{R}\right)$, is a ring and is equal to the maximal right quotient ring of $R$ [9, Corollary 2.31].

A module $M$ is called Hopfian if $M$ is not isomorphic to any of its proper factor modules (equivalently, every onto endomorphism of $M$ is 1-1). Anti-Hopfian modules are introduced by Hirano and Mogami [13]. Such modules are isomorphic to all their non-zero factor modules. A module $M$ is called uniserial if the lattice of submodules are linearly ordered. Anti-Hopfian modules are uniserial artinian.

Recall that a module $M$ is called coHopfian if it is not isomorphic to a proper submodule (equivalently, every 1-1 endomorphism of $M$ is onto). Varadarjan [22] dualized the concept of anti-Hopfian module and called it anti-coHopfian module. With slight modification we will call a non-zero module to be anti-coHopfian if it is isomorphic to all its non-zero submodules. A non-zero module $M$ is called uniform if 
the intersection of any two non-zero submodules is non-zero. We see an anti-coHopfian module is noetherian and uniform (see Lemma 3.1 below).

An $R$-module $M$ has cancellation property if for every $R$-modules $N$ and $T, M \oplus$ $N \cong M \oplus T$ implies $N \cong T$. Every module with semilocal endomorphism ring has cancellation property [16]. Since the endomorphism ring of a simple module is a division ring, it has cancellation property.

Unless otherwise specified, throughout this paper $R$ denotes an arbitrary ring and all modules are assumed to be unitary right modules. If $N$ is a submodule (resp. proper submodule) of $M$ we write $N \leq M$ (resp. $N<M$ ). Also, for a module $M, \oplus_{i=1}^{\infty} M$ stands for countably infinite direct sum of copies of $M$. If $N$ is a submodule of $M$ and $k>1, \oplus_{i=k}^{\infty} N=\oplus_{i=1}^{\infty} N_{i}$ is a submodule of $\oplus_{i=1}^{\infty} M$ with $N_{1}=N_{2}=\cdots=N_{k-1}=$ 0 and for $i \geq k N_{i}=N$.

\section{Basic and preliminary results}

As defined in the introduction, couniserial dimension is an ordinal valued number. The reader may refer to [21] regarding ordinal numbers. We begin this section with a lemma and a remark on the definition of couniserial dimension.

Lemma 3.1 An anti-coHopfian module is uniform noetherian.

Proof Since $M$ is isomorphic to each cyclic submodules, $M$ is cyclic and every submodule of $M$ is cyclic and so $M$ is noetherian. Thus $M$ has a uniform submodule, say $U$. Since $U \cong M, M$ is uniform.

Remark 3.2 We make the convention that a statement "c.u.dim $(M)=\alpha$ " will mean that the couniserial dimension of $M$ exists and equals $\alpha$. By the definition of couniserial dimension, if $M$ has couniserial dimension and $N$ is a submodule of $M$, then $N$ has couniserial dimension and c.u.dim $(N) \leq$ c.u.dim $(M)$. Moreover, if $M$ is not uniform and c.u.dim $(M)=$ c.u.dim $(N)$, where $N$ is a submodule of $M$, then $M$ $\cong N$. On the other hand, since every set of ordinal numbers has supremum, it follows immediately from the definition that $M$ has couniserial dimension if and only if for all submodules $N$ of $M$ with $N \varsubsetneqq M$, c.u.dim( $(N)$ is defined. In the latter case, if $\alpha$ $=\sup \{\mathrm{c} \cdot \operatorname{u} \cdot \operatorname{dim}(N) \mid N \leq M, N \nsubseteq M\}$, then c.u.dim $(M) \leq \alpha+1$.

The next proposition provides a working criterion for a module to have couniserial dimension.

Proposition 3.3 An $R$-module $M$ has couniserial dimension if and only if for every descending chain of submodules $M_{1} \geq M_{2} \geq \cdots$, there exists $n \geq 1$ such that $M_{n}$ is uniform or $M_{n} \cong M_{k}$ for all $k \geq n$.

Proof $(\Rightarrow)$ Let $M_{1} \geq M_{2} \geq \cdots$ be a descending chain of submodules of $M$. Put $\gamma=\inf \left\{\right.$ c.u.dim $\left.\left(M_{n}\right) \mid n \geq 1\right\}$. So $\gamma=$ c.u.dim $\left(M_{n}\right)$ for some $n \geq 1$. If $M_{n}$ is not uniform, then $M_{n} \cong M_{k}$ for all $k \geq n$, because $\gamma$ is infimum.

$(\Leftarrow)$ If $M$ does not have couniserial dimension, then $M$ is not uniform and so there exists a submodule $M_{1}$ of $M$ such that $M_{1} \not M M$ and $M_{1}$ does not have couniserial 
dimension, by the above remark. So there exists a submodule $M_{2}$ of $M_{1}$ such that $M_{2} \nsucceq M_{1}$ and $M_{2}$ does not have couniserial dimension. Continuing in this manner, we obtain a descending chain of submodules $M_{1} \geq M_{2} \geq \cdots$, such that for every $i \geq 1, M_{i}$ does not have couniserial dimension and $M_{i} \not M_{i+1}$, a contradiction. This completes the proof.

As a consequence, we have the following corollary.

Corollary 3.4 Every artinian module has couniserial dimension.

Lemma 3.5 If $M$ is an $R$-module and c.u.dim $(M)=\alpha$, then for any $0 \leq \beta \leq \alpha$, there exists a submodule $N$ of $M$ such that c.u.dim $(N)=\beta$.

Proof The proof is by transfinite induction on c.u.dim $(M)=\alpha$. The case $\alpha=1$ is clear. Let $\alpha>1$ and $0 \leq \beta<\alpha$, then, using Remark 3.2, there exists a submodule $K$ of $M$ such that $K \not M$ and $\beta \leq$ c.u.dim $(K)$. Now since $\beta \leq$ c.u.dim $(K)<\alpha$, by induction hypothesis, there exists a submodule $N$ of $K \operatorname{such}$ that c.u.dim $(N)=\beta$.

As a consequence we have the following.

Lemma 3.6 Every module with couniserial dimension has a uniform submodule.

In the next proposition we observe that every module of finite couniserial dimension has finite uniform dimension.

Lemma 3.7 Let $M$ be an $R$-module of finite couniserial dimension. Then $M$ has finite uniform dimension and $\mathrm{u} \cdot \operatorname{dim}(M) \leq \mathrm{c} \cdot \mathrm{u} \cdot \operatorname{dim}(M)$.

Proof The proof is by induction on c.u.dim $(M)=n$. The case $n=1$ is clear. Let $n>1$ and $N$ be a submodule of $M$ such that c.u.dim $(N)=n-1$. Thus by the inductive hypothesis, $N$ has finite uniform dimension. Put $m=\operatorname{u} \cdot \operatorname{dim}(N)$. If $N$ is not essential in $M$, then there exists a uniform submodule $U$ of $M$ such that $N \cap U=0$. Thus $N \oplus U$ is a submodule of $M$ of uniform dimension $m+1$. Then $(N \oplus U) \nsucceq N$ and so $n-1<$ c.u.dim $(N \oplus U) \leq n$. Thus $(N \oplus U) \cong M$, by Remark 3.2. This proves the lemma.

Example 3.8 There exist modules of infinite couniserial dimension but of finite uniform dimension. Take $M=\mathbb{Z}_{p^{\infty}} \oplus \mathbb{Z}_{p^{\infty}}$. Then $M$ is an artinian $\mathbb{Z}$-module of infinite couniserial dimension but of finite uniform dimension 2 .

In the following, we show that, in a special case, equality holds in the previous lemma.

Lemma 3.9 Let $M$ be an injective non-uniform $R$-module of finite couniserial dimension. Then c.u.dim $(M)=\mathrm{u} \cdot \operatorname{dim}(M)$ if and only if $M$ is a finitely generated semisimple module.

Proof $(\Leftarrow)$ is clear. $(\Rightarrow)$. Let c.u.dim $(M)=\mathrm{u} \cdot \operatorname{dim}(M)=m>1$. Then $M=E_{1} \oplus$ $\cdots \oplus E_{m}$, where $E_{i}$ are uniform injective modules. If $E_{1}$ is not simple then there exists a non-injective submodule $K$ of $E_{1}$. Thus $K \oplus E_{2} \oplus \cdots \oplus E_{m}$ is not isomorphic to $M$. But clearly c.u.dim $\left(K \oplus E_{2} \oplus \cdots \oplus E_{m}\right) \geq m$, a contradiction. This completes the proof. 
Note that the condition of being injective is necessary in the above proposition.

Example 3.10 We can see easily that for $M=\mathbb{Z} \oplus \mathbb{Z}$, c.u.dim $(M)=\mathrm{u} \cdot \operatorname{dim}(M)=2$ but $M$ is not semisimple. Also, the next lemma shows that there exists a module of finite uniform dimension without couniserial dimension.

The following lemma shows that the direct sum of two uniform modules may not have couniserial dimension.

Lemma 3.11 Let $D$ be a domain and $S$ be a simple $D$-module. If $S \oplus D$ as $D$-module has couniserial dimension, then $D$ is a principal right ideal domain.

Proof Let $I$ be a non-cyclic right ideal of $D$. Choose a non-zero element $x \in I$. Set $J_{1}=x R$ which is isomorphic to $D$. Thus there exists a right ideal $J_{2}$ of $D$ such that $J_{2} \cong I$ and $J_{2} \leq J_{1}$. Now let $J_{3}$ be a cyclic right ideal contained in $J_{2}$ and by continuing this manner we have a descending chain $J_{1} \geq J_{2} \geq \cdots$ of right ideals of $D$ where for each odd integer $i, J_{i}$ is cyclic and for each even integer $i, J_{i}$ is not cyclic. Now consider the descending chain $S \oplus J_{1} \geq S \oplus J_{2} \geq \cdots$ of submodules of $S \oplus D$. Since $S$ has cancellation property and for each $i, S \oplus J_{i}$ is not uniform, by using Proposition 3.3, we see that, for some $n, J_{n} \cong J_{n+1}$, a contradiction. Thus $D$ is a principal right ideal domain.

Remark 3.12 (1) The simple module $S$ in the statement of the Lemma 3.11 can be replaced by any cancellable module. Indeed it follows from Theorem 4.10, proved later that, if the maximal right quotient ring $Q$ of a domain $D$ has couniserial dimension as $D$-module, then $Q_{D}$ has cancellation property and so if $Q \oplus D$ as $D$-module has couniserial dimension, $D$ must be right principal ideal domain.

(2) Also, since a Dedekind domain has cancellation property, similar proof shows that if $D$ is a Dedekind domain which is not right principal ideal domain, then $D \oplus D$ does not have couniserial dimension. This example shows that even direct sum of a uniform module with itself may not have couniserial dimension.

The definition of addition two ordinal numbers can be given inductively. If $\alpha$ and $\beta$ are two ordinal numbers then $\alpha+0=\alpha, \alpha+(\beta+1)=(\alpha+\beta)+1$ and if $\gamma$ is a limit ordinal then $\alpha+\gamma$ is the limit of $\alpha+\beta$ for all $\beta<\gamma$ (see [21]).

Lemma 3.13 (See [21, Theorem 7.10]) For ordinal numbers $\alpha, \beta$ and $\gamma$, we have the following:

(1) If $\alpha<\beta$, then $\gamma+\alpha<\gamma+\beta$.

(2) If $\alpha<\beta$, then $\alpha+\gamma \leq \beta+\gamma$.

We call an $R$-module $M$ fully coHopfian if every submodule of $M$ is coHopfian. Note that artinian modules are fully coHopfian. If $I$ is the set of prime numbers, then $\oplus_{p \in I} \mathbb{Z}_{p}$ is an example of fully coHopfian $\mathbb{Z}$-module that it is not artinian.

Proposition 3.14 Let $M=M_{1} \oplus M_{2}$ be a fully coHopfian $R$-module with couniserial dimension. Then c.u.dim $(M) \geq$ c.u.dim $\left(M_{1}\right)+$ c.u.dim $\left(M_{2}\right)$. 
Proof We may assume $M_{1}, M_{2} \neq 0$. We use transfinite induction on c.u.dim $\left(M_{2}\right)=$ $\alpha$. Since $M_{1} \nsucceq M$, c.u. $\operatorname{dim}(M) \geq$ c.u.dim $\left(M_{1}\right)+1$. So the case $\alpha=1$ is clear. Thus, suppose $\alpha>1$ and for every right $R$-module $L$ of couniserial dimension less than $\alpha$, c.u.dim $\left(M_{1} \oplus L\right) \geq$ c.u.dim $\left(M_{1}\right)+$ c.u.dim $(L)$. There are two possibilities: $\alpha$ is a successor ordinal, or a limit ordinal. Suppose first that $\alpha$ is a successor ordinal, then there exists an ordinal number $\gamma$ such that c.u.dim $\left(M_{2}\right)=\gamma+1$. Using Lemma 3.5, there exists a non-zero submodule $K$ of $M_{2}$ such that c.u.dim( $\left.K\right)=\gamma<\alpha$. So by the induction hypothesis

$$
\text { c.u.dim }\left(M_{1}\right)+\gamma=\text { c.u.dim }\left(M_{1}\right)+\text { c.u.dim }(K) \leq \text { c.u.dim }\left(M_{1} \oplus K\right) \text {. }
$$

Using our assumption and Remark 3.2, we have c.u.dim $\left(M_{1} \oplus K\right)<$ c.u.dim $(M)$ and hence c.u.dim $\left(M_{1}\right)+$ c.u.dim $\left(M_{2}\right) \leq$ c.u.dim $(M)$.

If $\alpha$ is a limit ordinal and $1 \leq \beta<\alpha$, then by Remark 3.2, there exists a nonzero submodule $K$ of $M_{2}$ such that $\beta \leq$ c.u.dim( $\left.K\right)$. Then by induction hypothesis c.u.dim $\left(M_{1}\right)+\beta \leq$ c.u.dim $\left(M_{1}\right)+$ c.u.dim $(K) \leq$ c.u.dim $\left(M_{1} \oplus K\right)<$ c.u.dim $(M)$. Therefore c.u.dim $\left(M_{1}\right)+\alpha=\sup \left\{\right.$ c.u.dim $\left.\left(M_{1}\right)+\beta \mid \beta<\alpha\right\} \leq$ c.u.dim $(M)$.

The condition fully coHopfian of Proposition 3.14 is necessary.

Example 3.15 For the $\mathbb{Z}$-modules $M=\oplus_{i=1}^{\infty} \mathbb{Z}_{p}$, and $L=\mathbb{Z}_{p}$, we have $M \cong M \oplus L$. One can see c.u.dim $(M)=\omega$ and so c.u.dim( $(M) \nsupseteq$ c.u.dim $(M)+$ c.u.dim $(L)$. Also, in general, we don't have the equality in Proposition 3.14. Consider the $\mathbb{Z}$-module $M=\mathbb{Z}_{2} \oplus \mathbb{Z}_{4}$. Then, $M$ is fully coHopfian and $3=$ c.u.dim $(M)>$ c.u.dim $\left(\mathbb{Z}_{2}\right)+$ c.u.dim $\left(\mathbb{Z}_{4}\right)$.

Here we prove another result on fully coHopfian module:

Proposition 3.16 Let $M$ be an $R$-module and $N$ be a cancellable module (for example a simple module) such that $N \oplus M$ has couniserial dimension. If $M$ is fully coHopfian, then $M$ is artinian.

Proof Let $M$ be fully coHopfian and let $M_{1} \geq M_{2} \geq \cdots$ be a descending chain of submodules of $M$. Then $N \oplus M_{1} \geq N \oplus M_{2} \geq \cdots$ is a descending chain of submodules of $N \oplus M$ and so for some $n, M_{i} \cong M_{n}$ for each $i \geq n$. Now since $M$ is fully coHopfian, we have $M_{i}=M_{n}$ for each $i \geq n$.

Let us recall the definition of uniserial dimension [20].

Definition 3.17 In order to define uniserial dimension for modules over a ring $R$, we first define, by transfinite induction, classes $\zeta_{\alpha}$ of $R$-modules for all ordinals $\alpha \geq 1$. To start with, let $\zeta_{1}$ be the class of non-zero uniserial modules. Next, consider an ordinal $\alpha>1$; if $\zeta_{\beta}$ has been defined for all ordinals $\beta<\alpha$, let $\zeta_{\alpha}$ be the class of those $R$-modules $M$ such that, for every submodule $N<M$, where $M / N \not M$, we have $M / N \in \bigcup_{\beta<\alpha} \zeta_{\beta}$. If an $R$-module $M$ belongs to some $\zeta_{\alpha}$, then the least such $\alpha$ is the uniserial dimension of $M$, denoted u.s.dim( $M)$. For $M=0$, we define u.s.dim $(M)=0$. If $M$ is non-zero and $M$ does not belong to any $\zeta_{\alpha}$, then we say that "u.s.dim $(M)$ is not defined," or that " $M$ has no uniserial dimension." 
Remark 3.18 Note that, in general, there is no relation between the existence of the uniserial dimension and the existence of the couniserial dimension of a module. For example, the polynomial ring in infinite number of commutative indeterminates over a field $k, R=k\left[x_{1}, x_{2}, \ldots\right]$ has this property that c.u.dim $\left(R_{R}\right)=1$ but $R_{R}$ does not have uniserial dimension (see [6, Remark 2.3]). It follows by the definition that a semisimple module $M$ has uniserial dimension if and only if $M$ has couniserial dimension, in which case u.s.dim $(M)=\alpha$ if and only if c.u.dim $(M)=\alpha$. Furthermore a semisimple module $M$ has couniserial dimension if and only if $M$ is a finite direct sum of homogeneous semisimple modules (see [20, Proposition 1.18]).

Using the above remark we have the following interesting results.

Corollary 3.19 All right semisimple modules over a ring $R$ have couniserial dimension if and only if there exist only finitely many non-isomorphic simple right $R$-modules.

Lemma 3.20 Let $M$ be a finitely generated cancellable module. Then:

(1) If $\oplus_{i=1}^{\infty} E(M)$ has couniserial dimension, then $M$ is injective.

(2) Suppose that $M$ is simple. Then $\oplus_{i=1}^{\infty} E(M)$ has couniserial dimension if and only if $M$ is injective.

Proof (1) Consider the descending chain

$$
M \oplus\left(\oplus_{i=2}^{\infty} E(M)\right) \geq M^{(2)} \oplus\left(\oplus_{i=3}^{\infty} E(M)\right) \geq \cdots
$$

of submodules of $\oplus_{i=1}^{\infty} E(M)$ where $M^{(n)}=\oplus_{i=1}^{\infty} M_{i}$ with $M_{1}=\cdots=M_{n}=M$ and for each $i>n, M_{i}=0$. Then, by Proposition 3.3, there exists $n \geq 1$ such that

$$
M^{(n)} \oplus\left(\oplus_{i=n+1}^{\infty} E(M)\right) \cong M^{(n+1)} \oplus\left(\oplus_{i=n+2}^{\infty} E(M)\right)
$$

and so $\oplus_{i=n+1}^{\infty} E(M) \cong M \oplus\left(\oplus_{i=n+2}^{\infty} E(M)\right)$, because $M$ is cancellable . Since $M$ is finitely generated, there exists a right module $L$ such that for some $k, E(M)^{k}$ $\cong M \oplus L$. This shows $M$ is injective.

(2) It is clear by part 1 and the statement in Remark 3.18.

\section{Main results}

In this section we use our basic results to prove the main results.

Proposition 4.1 Let $M_{R}$ be an injective module and $N_{R}$ be a cancellable module over a commmutative ring $R$ (for example a simple module) such that $N \oplus M$ has couniserial dimension. Then $M$ is $\Sigma$-injective.

Proof According to [15, Theorem 6.17], it is enough to show that $R$ satisfies the ascending chain condition on ideals of $R$ that are annihilators of subsets of $M$. Let 
$I_{1} \leq I_{2} \leq \cdots$ be a chain of such annihilator ideals. Then for each $i, M_{i}=\operatorname{ann}_{M}\left(I_{i}\right)$ is a submodule of $M$ and so we have descending chain $N \oplus M_{1} \geq N \oplus M_{2} \geq \cdots$ of submodules of $N \oplus M$. Then there exists a positive integer $n$ such that $M_{n} \cong M_{i}$ for all $i \geq n$. Thus $\operatorname{ann}\left(M_{i}\right)=\operatorname{ann}\left(M_{n}\right)$. Therefore for each $i \geq n, I_{i}=I_{n}$.

Remark 4.2 If $S$ is a simple module, then $S \oplus S$ has couniserial dimension and $S$ is also a cancellable module. Thus, the above result provides another proof for the fact that commutative V-rings (i.e, von Neumann regular rings) are $\Sigma-\mathrm{V}$-ring. Now if $R$ is a right V-ring that is not right $\Sigma$-V-ring, see [15, Example, page 60], then there exists a simple right $R$-module $S$ which is not $\Sigma$-injective. This shows that the commutativity condition cannot be dropped in the above proposition.

The next result shows that if a module has countable couniserial dimension then it can be decomposed into indecomposable modules.

Theorem 4.3 For an $R$-module $M$, if c.u.dim $(M) \leq \omega$, then $M$ has indecomposable decomposition.

Proof The proof is by induction on c.u.dim $(M)=\alpha$. The case $\alpha=1$ is clear. If $\alpha>1$ and $M$ is not indecomposable, then $M=N_{1} \oplus N_{2}$, where $N_{1}$ and $N_{2}$ are non-zero submodules of $M$. If c.u.dim $\left(N_{i}\right)<\mathrm{c} \cdot \mathrm{u} \cdot \operatorname{dim}(M), i=1,2$, then by induction hypothesis $M$ has indecomposable decomposition. If not, for definiteness let c.u.dim $\left(N_{1}\right)=$ c.u.dim $(M)$. Then $M \cong N_{1}$, by Remark 3.2. Thus it contains an infinite direct sum of uniform modules, say $\oplus_{i=1}^{\infty} K_{i}$. Clearly, c.u.dim $\left(\oplus_{i=1}^{\infty} K_{i}\right) \geq \omega$. Thus we have $M \cong \oplus_{i=1}^{\infty} K_{i}$.

Remark 4.4 We do not know whether the above proposition holds for a module of arbitrary couniserial dimension. For infinite countable couniserial dimension one can show under some condition that the module can be represented as a direct sum of uniform modules.

Recall that a module $M$ is called Dedekind finite if $M$ is not isomorphic to any proper direct summand of itself. Clearly, every direct summand of a Dedekind finite module is a Dedekind finite module. Obviously, a Hopfian module is Dedekind finite. Since all finitely generated modules over a commutative ring are Hopfian (see [10]), they provide examples of Dedekind finite modules.

Theorem 4.5 If $M$ is a Dedekind finite module with couniserial dimension, then $M$ has finite indecomposable decomposition.

Proof The proof is by induction on c.u.dim $(M)=\alpha$. The case $\alpha=1$ is clear. Let $\alpha>1$ and every Dedekind finite module with c.u.dim less than $\alpha$ be decomposed to finitely many indecomposable modules. If $M$ is not indecomposable, then $M=$ $M_{1} \oplus M_{2}$. Since $M_{i} ¥ M$, using Remark 3.2, c.u.dim $\left(M_{i}\right)<$ c.u.dim $(M)$ and so, by induction hypothesis, $M_{i}$ have finite indecomposable decomposition. This completes the proof.

A ring $R$ is called a von Neumann regular ring if for each $x \in R$, there exists $y \in R$ such that $x y x=x$, equivalently, every principal right ideal is a direct summand. $R$ is unit regular ring if for each $x \in R$, there exists a unit element $u \in R$ such that $x=x u x$. As a consequence of the above theorem we have the following corollary. 
Corollary 4.6 Every Dedekind finite von Neumann regular ring (in particular, unit regular rings) with couniserial dimension is semisimple artinian.

A ring $R$ is called a PWD (piecewise domain) if it possesses a complete set $\left\{e_{i} \mid 0\right.$ $\leq i \leq n\}$ of orthogonal idempotents such that $x y=0$ implies $x=0$ or $y=0$ whenever $x \in e_{i} R e_{k}$ and $y \in e_{k} R e_{j}$. Note that the definition is left-right symmetric and all $e_{i} R e_{i}$ are domain, see [12].

An element $x$ of $R$ is called regular if its right and left annihilators are zero.

Proposition 4.7 Let $R$ be a semiprime right Goldie ring with couniserial dimension. If $u . \operatorname{dim}\left(R_{R}\right)=n$, then $R$ has a decomposition into $n$ uniform modules. In particular, it is a piecewise domain.

Proof We can assume that $n>1$. Let $I_{1}=U_{1} \oplus \cdots \oplus U_{n}$ be an essential right ideal of $R$. Then, by [8, Proposition 6.13], $I_{1}$ contains a regular element $x$ and thus $J_{1}=x R$ is a right ideal of $R$ which is $R$-isomorphic to $R$. So u.dim $\left(J_{1}\right)=n$ and it contains an essential right ideal $I_{2}$ of $R$ such that it is a direct sum of $n$ uniform right ideals. By continuing in this manner we obtain a descending chain $I_{1} \geq J_{1} \geq I_{2} \geq \cdots$ of right ideals of $R$ such that $I_{i}$ are direct sum of $n$ uniform right ideals and $J_{i}$ are isomorphic to $R$. Since $R$ has couniserial dimension, for some $n, I_{n} \cong R$. The last statement follows from [12, Pages 2-3]. This completes the proof.

Remark 4.8 There exists an example of simple noetherian ring of uniform dimension 2 which has no non-trivial idempotents (c.f. [18, Example 7.16, page 441]). So by the above proposition this provides an example of prime right Goldie ring without couniserial dimension.

Lemma 4.9 Let $R$ be a right non-singular ring with maximal right quotient ring $Q$. Let $M$ be a $Q$-module. If $M$ is non-singular $R$-module, such that $M_{R}$ has couniserial dimension, then $M_{Q}$ has couniserial dimension.

Proof Let $M \geq M_{1} \geq M_{2} \geq \cdots$ be a descending chain of $Q$-submodules of $M$. So it is a descending chain of $R$-submodules of $M$ and thus, for some $n, M_{n}$ is uniform $R$ module or $M_{n} \cong M_{i}$ as $R$-modules for all $i \geq n$. If $M_{n}$ is uniform $R$-module, then it is also uniform $Q$-module. So let $M_{n} \cong M_{i}$ as $R$-modules and let $\varphi_{i}$ be this isomorphism. If $q \in Q$ and $t \in M_{n}$ there exists an essential right ideal $E$ of $R$ such that $q E \leq R$. So $\varphi_{i}(t q E)=\varphi_{i}(t q) E$ and also $\varphi_{i}(t q E)=\varphi_{i}(t) q E$. Then $\varphi_{i}(t q) E=\varphi_{i}(t) q E$. Since $Q$ is right non-singular, $\varphi_{i}(t q)=\varphi_{i}(t) q$. Thus $\varphi_{i}$ is a $Q$-isomorphism. This completes the proof.

A ring $R$ is semiprime (prime) right Goldie ring if and only if its classical right quotient ring is semisimple (simple) artinian ring [9, Theorems 3.35 and 3.36]. Semiprime right Goldie rings are non-singular. A semiprime right non-singular ring $R$ is semiprime right Goldie ring if and only if $\operatorname{ud} \operatorname{dim}\left(R_{R}\right)$ is finite, [9, Theorem 3.17]. Recall that a right full linear ring is the ring of all linear transformations (written on the left) of a right vector space over a division ring. If the dimension of the vector space is finite, a right full linear ring is exactly a simple artinian ring. 
Theorem 4.10 Let $R$ be a semiprime right non-singular ring with maximal right quotient ring, $Q$. If $Q$ as an $R$-module has couniserial dimension, then $R$ is a semiprime right Goldie ring which is a finite product of prime Goldie rings, each of which is a piecewise domain.

Proof It is enough to show that $R$ has finite uniform dimension. Since $Q_{R}$ has couniserial dimension, $R_{R}$ has couniserial dimension and so every right ideal of $R$ has couniserial dimension. Thus Lemma 3.6 implies that every right ideal contains a uniform submodule. Now by [9, Theorem 3.29] the maximal right quotient ring of $R$ is a product of right full linear rings, say $Q=\prod_{i \in I} Q_{i}$, where $Q_{i}$ are right full linear rings. Note that since $R_{R}$ is right non-singular, $Q_{R}$ is also non-singular and so, using Lemma 4.9, $Q_{Q}$ has couniserial dimension. At first we claim each $Q_{i}$ is endomorphism ring of a finite dimensional vector space. Assume the contrary. Then $Q_{j}$ is the endomorphism ring of an infinite dimensional vector space, for some $j$. Thus $Q_{j} \cong Q_{j} \times Q_{j}$ and so if $\iota: Q_{j} \longrightarrow Q$ be the canonical embedding, then $\iota\left(Q_{j}\right)$ is a right ideal of $Q$ and there exists a $Q$-isomorphism $Q \cong \iota\left(Q_{j}\right) \times Q$. Then there exist right ideals $T_{1}$ and $T$ of $Q$ such that $Q=T_{1} \oplus T, T_{1}$ and $Q$ are isomorphic as $Q$-modules and $T \cong \iota\left(Q_{j}\right)$ as $Q$-module. Because $Q_{j}$ is the endomorphism ring of an infinite dimensional vector space, it has a right ideal which is not principal, for example its socle. So $\iota\left(Q_{j}\right)$ and thus $T$ contains a non-cyclic right ideal of $Q$ and thus since $T \cong Q / T_{1}$, there exists a non-cyclic right ideal of $Q$, say $K_{1}$ such that $Q \geq K_{1} \geq T_{1}$. Now $T_{1}$ is isomorphic to $Q$. So we can have a descending chain $Q>K_{1}>T_{1}>K_{2}>T_{2}>\cdots$ of right ideals of $Q$ such that $T_{i}$ are cyclic but $K_{i}$ are not cyclic. This is a contradiction. So all $Q_{i}$ are endomorphism ring of finite dimensional vector spaces. Now to show $R$ is semiprime right Goldie ring it is enough to show that the index set $I$ is finite. If $I$ is infinite, there exist infinite subsets $I_{1}$ and $I_{2}$ of $I$ such that $I=I_{1} \cup I_{2}$. and $I_{1} \cap I_{2}$ is empty. Let $T_{1}=\prod_{i \in I} N_{i}$ such that $N_{i}=Q_{i}$ for all $i \in I_{1}$ and $N_{i}=0$ for all $i \in I_{2}$. Similarly let $T=\prod_{i \in I} M_{i}$ such that $M_{i}=Q_{i}$ for all $i \in I_{2}$ and $M_{i}=0$ for all $i \in I_{1}$. Then $T_{1}$ and $T$ are right ideals of $Q$ and $Q=T_{1} \oplus T$. T contains a right ideal of $Q$ which is not cyclic, for example $\bigoplus_{i \in I} M_{i}$. Since $T \cong Q / T_{1}$, there exists a non-cyclic right ideal $K_{1}$ of $Q$ such that $Q \geq K_{1} \geq T_{1}$. Note that $T_{1}$ is a cyclic $Q$-module and because $I_{1}$ is infinite, the structure of $T_{1}$ is similar to that of $Q$. We can continue in this manner and find a descending chain of right ideals of $Q$ such that $K_{i}$ are non cyclic $Q$-modules and $T_{i}$ are cyclic $Q$ modules, which is a contradiction. Therefore $I$ is finite and $R$ must have finite uniform dimension. This shows $R$ is semiprime right Goldie ring and so Proposition 4.7 and [12, Corollary 3] imply that it is a direct sum of prime right Goldie rings.

We may point out that unlike a semiprime ring with right Krull dimension, a semiprime ring with couniserial dimension need not be a right Goldie ring. See Dubrovin [5] that contains an example of a primitive uniserial ring with non-zero nilpotent elements.

Next we show that the converse of the above theorem is not true, in general. In fact we show that there exists a prime right Goldie ring $R$ such that c.u.dim $\left(R_{R}\right)=2$ and $Q_{R}$ does not have couniserial dimension. We need the following lemma to give the example. 
Lemma 4.11 For an ordinal number $\alpha$, being of couniserial dimension $\alpha$ is a Morita invariant property for modules.

Proof This is clear by the definition of couniserial dimension and [1, Proposition 21.7].

Example 4.12 Here we give an example of a prime right Goldie ring $R$ with maximal right quotient ring $Q$ such that $Q_{R}$ does not have couniserial dimension. Take $R=$ $M_{2}(\mathbb{Z})$, the $2 \times 2$ matrix ring over $\mathbb{Z}$. Then $R$ is a prime right Goldie ring with maximal right quotient ring $Q=M_{2}(\mathbb{Q})$. Note that under the standard Morita equivalent between the ring $\mathbb{Z}$ and $R=M_{2}(\mathbb{Z})$, see [17, Theorem 17.20 ], $R$ corresponds to $\mathbb{Z} \oplus \mathbb{Z}$ and so using the above lemma $R$ has couniserial dimension 2 . If $\left\{p_{i} \mid i \geq 1\right\}$ is the set of all prime numbers, then $\mathbb{Q} / \mathbb{Z}=\sum_{i=1}^{\infty} K_{i} / \mathbb{Z}$, where $K_{i}=\left\{m / p_{i}^{n} \mid n \geq 0\right.$ and $m \in \mathbb{Z}$ \}. Then take $Q_{n}=\sum_{i=n}^{\infty} K_{i}$. Then $M_{2}\left(Q_{1}\right) \geq M_{2}\left(Q_{2}\right) \geq \cdots$ is a descending chain of $R$-submodules of $Q$ which are not uniform $R$-modules. Assume that for some $n, M_{2}\left(Q_{n}\right) \cong M_{2}\left(Q_{n+1}\right)$ with an $R$-isomorphism $\phi$. Let $\phi\left(\begin{array}{ll}1 & 0 \\ 0 & 1\end{array}\right)=$ $\left(\begin{array}{ll}m_{1} / t_{1} & m_{2} / t_{2} \\ m_{3} / t_{3} & m_{4} / t_{4}\end{array}\right)$, where $m_{i} / t_{i} \in Q_{n+1}$. Suppose that $j \geq 1$ and $\phi\left(\begin{array}{ll}1 / p_{n}^{j} & 0 \\ 0 & 1 / p_{n}^{j}\end{array}\right)=$ $\left(\begin{array}{ll}m_{1, j} / t_{1, j} & m_{2, j} / t_{2, j} \\ m_{3, j} / t_{3, j} & m_{4, j} / t_{4, j}\end{array}\right)$, where $p_{n}$ does not odd non of $t_{i, j}$ for all $1 \leq i \leq 4$. Then since $\phi$ is additive, we can easily see that $\left(\begin{array}{ll}m_{1, j} p_{n}^{j} / t_{1, j} & m_{2, j} p_{n}^{j} / t_{2, j} \\ m_{3, j} p_{n}^{j} / t_{3, j} & m_{4, j} p_{n}^{j} / t_{4, j}\end{array}\right)=\left(\begin{array}{ll}m_{1} / t_{1} & m_{2} / t_{2} \\ m_{3} / t_{3} & m_{4} / t_{4}\end{array}\right)$ and this implies that $p_{n}^{j} \mid m_{i}$ for all $j \geq 1$ and $0 \leq i \leq 4$ and so $m_{i}=0$, a contradiction. So $Q_{R}$ does not have couniserial dimension.

\section{Some applications}

A right $R$-module $M$ which has a composition series is called a module of finite length. A right $R$-module $M$ is of finite length if and only if $M$ is both artinian and noetherian. The length of a composition series of $M_{R}$ is said to be the length of $M_{R}$ and is denoted by length $(M)$. Clearly, by Corollary 3.4, a module of finite length has couniserial dimension. The next result shows a relation between couniserial dimension of a finite length module $M$ and length $(M)$.

Proposition 5.1 Let $M$ be a right $R$-module of finite length. Then the following statements hold:

(1) If $N$ is a submodule of $M$, then c.u.dim $(M / N) \leq$ c.u.dim( $M)$.

(2) c.u.dim $(M) \leq$ length $(M)$.

Proof (1) The proof is by induction on $n$, where length $(M)=n$. The case $n=1$ is clear. Now, let $n>1$ and assume that the assertion is true for all modules with length less than $n$. If $N$ is a non-zero submodule of $M$, then the length $(M / N)<n$. Thus for every proper submodule $K / N$ of $M / N$, by induction, c.u.dim $(K / N) \leq$ c.u.dim $(K)<$ c.u.dim $(M)$. Now, Remark 3.2 implies that c.u.dim $(M / N) \leq$ c.u.dim $(M)$. 
(2) The proof is by induction on length $(M)=n$. The case $n=1$ is clear. Now if $n>1$ and $K$ is a proper submodule of $M$, then, by assumption, c.u.dim( $K) \leq$ length $(K)<$ length $(M)$. Thus by $\operatorname{Remark} 3.2$, c.u.dim $(M) \leq \operatorname{length}(M)$.

Recall that an $R$-module $M$ is called co-semisimple if every simple $R$-module is $M$-injective, or equivalently, $\operatorname{Rad}(M / N)=0$ for every submodule $N \leq M$ (See [23, Theorem 23.1]). The next proposition gives a condition as to when a module of finite length is semisimple. It may be of interest to state that for the finite length $\mathbb{Z}$-module $\mathbb{Z}_{4}, \oplus_{i=1}^{\infty} \mathbb{Z}_{4}$ does not possess couniserial dimension.

Proposition 5.2 Let $M$ be a non-zero right $R$-module of finite length. Then $M$ is a semisimple $R$-module if and only if for every submodule $N$ of $M$ the right $R$-module $\oplus_{i=1}^{\infty} M / N$ has couniserial dimension.

Proof $(\Rightarrow)$ c.f. Remark 3.18.

$(\Leftarrow)$ For every submodule $N$ of $M$ the right $R$-module $\oplus_{i=1}^{\infty} M / N$ has couniserial dimension. Clearly, this also holds for any factor module of $M$. We will proof the result by induction on the length $(M)=n$. The case $n=1$ is clear. Now assume that $n>1$ and the result is true for all modules of length less than $n$. Let $K$ be a nonzero submodule of $M$. Since length $(M / K)<n$, by the inductive hypothesis, $M / K$ is semisimple. Therefore, for every non-zero submodule $K$ of $M, \operatorname{Rad}(M / K)=0$. If $\operatorname{Rad}(M)=0$, then $M$ is co-semisimple. Let $S$ be a simple submodule of $M$. Consider the exact sequence $0 \longrightarrow S \longrightarrow M \longrightarrow M / S \longrightarrow 0$ which splits, because $M$ is co-semisimple. Therefore, $M$ is semisimple. Next suppose that $\operatorname{Rad}(M) \neq 0$. Let $S$ be a simple submodule of $M$. Because by the above $\operatorname{Rad}(M / S)=0$, we obtain $\operatorname{Rad}(M) \leq S$. This implies $\operatorname{Rad}(M)=S$ and so $M$ has only one simple submodule. Thus $\operatorname{Rad}(M)=\operatorname{soc}(M)=S$ is a simple module. Suppose that $M$ is not semisimple. Let $N$ be a maximal submodule of $M$. Then for every submodule $K \leq N<M, \oplus_{i=1}^{\infty} N / K$ is a submodule of $\oplus_{i=1}^{\infty} M / K$ and thus $\oplus_{i=1}^{\infty} N / K$ has couniserial dimension. Since length $(N)<n$, we conclude that $N$ is semisimple. Thus $N=\operatorname{soc}(M)=\operatorname{Rad}(M)$ is a simple module and so $M$ is of length 2 .

Now consider the descending chain

$$
N \oplus\left(\oplus_{i=2}^{\infty} M\right)>N^{(2)} \oplus\left(\oplus_{i=3}^{\infty} M\right)>\cdots
$$

of submodules of $\oplus_{i=1}^{\infty} M$. Using Proposition 3.3, there exists $k \geq 1$ such that $N^{(k)} \oplus$ $\left(\oplus_{i=k+1}^{\infty} M\right) \cong N^{(k+1)} \oplus\left(\oplus_{i=k+2}^{\infty} M\right)$. Since $N^{(k+1)}$ is finitely generated, there exist $m \geq 0$ and an $R$-module $T$, such that $N^{(k)} \oplus M^{m} \cong N^{(k+1)} \oplus T$. $N$ is simple and so it has cancellation property and thus $M^{m} \cong N \oplus T$. This implies $\operatorname{Rad}(T)$ is semisimple of length $m$ and length $(\operatorname{soc}(T))=m-1$, a contradiction.

Recall that a ring $R$ is called right bounded if every essential right ideal contains a two-sided ideal which is essential as a right ideal. A ring $R$ is called right fully bounded if every prime factor ring is right bounded. A right noetherian right fully bounded ring is commonly abbreviated as a right FBN ring. Clearly all commutative noetherian rings are example of right FBN rings. Finite matrix rings over commutative noetherian rings are a large class of right FBN rings which are not commutative. In 
[14, Theorem 2.11], Hirano and et.al. showed that a right FBN ring $R$ is semisimple if and only if every right module of finite length is semisimple. As a consequence of the above proposition we have:

Corollary 5.3 A right FBN ring $R$ is semisimple if and only if for every finite length module $M$, the module $\oplus_{i=1}^{\infty} M$ has couniserial dimension.

Proposition 5.4 Let $P$ be an anti-coHopfian projective right $R$-module. If $\oplus_{i=1}^{\infty} E(P)$ has couniserial dimension, then $P$ is injective.

Proof By part 1 of Lemma 3.20 it is enough to show $P$ has cancellation property. Let $M=P \oplus B \cong P \oplus B^{\prime}$. So there exist submodules $P^{\prime}$ and $C$ of $M$ such that $M=P \oplus B=P^{\prime} \oplus C$ and $P^{\prime} \cong P$ and $C \cong B^{\prime}$. If $p_{1}$ is the projection map from $M=P \oplus B$ onto $P$ with kernel $B$. Then considering the restriction of $p_{1}$ to $C$ we have an exact sequence $0 \longrightarrow C \cap B \longrightarrow C \longrightarrow I \longrightarrow 0$, such that $I$ is a submodule of $P$. Note that every submodule of $P$ is projective, because it is anti-coHopfian. So $I$ is projective and thus $C \cong(C \cap B) \oplus I$. Similarly by considering the map $p_{2}$ from $M=P^{\prime} \oplus C$ to $P^{\prime}$ we have $B \cong C \cap B \oplus J$ for some submodule $J$ of $P^{\prime}$. Since $J \cong I \cong P$, we have $B \cong C$ and so $B \cong B^{\prime}$. Then $P$ has cancellation property.

As a consequence of the above proposition we have the following corollary:

Corollary 5.5 Let $R$ be a principal right ideal domain with maximal right quotient ring $Q$ (which is a division ring). If the right $R$-module $\oplus_{i=1}^{\infty} Q$ has couniserial dimension, then $R=Q$.

We need the following lemmas to prove the next theorem. Using Proposition 3.3 we can see that:

Lemma 5.6 Let $I$ be a two sided ideal of $R$ and $M$ be an $R / I$-module. If $M$ as $R$ module has couniserial dimension, then $M$ as $R / I$-module has couniserial dimension.

Lemma 5.7 If all finitely generated right modules have couniserial dimension, then every right module contains a noetherian uniform module.

Proof By Lemma 3.1 it is enough to show that every cyclic module contains an anti-coHopfian module. Let $M$ be a non-zero cyclic right module which does not contain anti-coHopfian module and let $S$ be a simple module. $M$ is not anti-coHopfian, then $M$ has a non-zero submodule $M_{1} ¥ M$ and $M_{1}$ has a non-zero submodule $M_{2}$ such that $M_{2} \nsubseteq M_{1}$. By continuing in this manner we have a descending chain $S \oplus M \geq S \oplus M_{1} \geq S \oplus M_{2} \geq \cdots$ of submodules of $S \oplus M$. Since $S \oplus M$ is finitely generated, by Proposition 3.3, $S \oplus M_{n} \cong S \oplus M_{n+1}$ for some $n$. This implies that $M_{n} \cong M_{n+1}$ for some $n$, because $S$ is cancellable and this is a contradiction.

Theorem 5.8 For a ring $R$ the following are equivalent.

(1) $R$ is a semisimple artinian ring.

(2) All right $R$-modules have couniserial dimension.

(3) All left $R$-modules have couniserial dimension. 
(4) All right $R$-modules have uniserial dimension.

(5) All left $R$-modules have uniserial dimension.

Proof For equivalence of (1), (4) and (5) refer [20, Theorem 2.6].

$(1) \Rightarrow(2)$. This is clear by Corollary 3.19 .

$(2) \Rightarrow(1)$. At first we show $R$ satisfies ascending chain condition on two sided deals. Let $I_{1} \leq I_{2} \leq \cdots$ be a chain of ideals of $R$. Since the right module $\oplus_{i=1}^{\infty} R / I_{i}$ has couniserial dimension, there exists $n$ such that, for each $j \geq n, \oplus_{i=n}^{\infty} R / I_{i} \cong$ $\oplus_{i=j}^{\infty} R / I_{i}$. Thus they have the same annihilators and so for each $j \geq n, I_{n}=I_{j}$. Suppose $R$ is a non-semisimple ring. By Lemma 5.6 every module over a factor ring of $R$ also has couniserial dimension. Thus by invoking the ascending chain condition on two sided ideals we may assume $R$ is not semisimple artinian but every factor ring of $R$ is semisimple artinian. Using Lemma 3.20, $R$ is a right V-ring. First let us assume that $R$ is primitive. So, by Theorem 4.10, $R$ is a prime right Goldie ring. By [4, Theorem 5.16], a prime right V-ring right Goldie is simple. By Lemma 5.7, $R$ has a right noetherian uniform submodule and so using [8, Corollary 7.25], $R$ is right noetherian. Now we show that $R$ is Morita equivalent to a domain. By [7, lemma 5.12], the endomorphism ring of every uniform right ideal of a prime right Goldie ring is a right Ore domain. So by [11, Theorem 1.2], it is enough to show that $R$ has a uniform projective generator $U$. Let us assume that $R$ is not uniform and u.dim $(R)=n$ and let $U$ be a uniform right ideal of $R$. By [8, Corollary 7.25], $U^{n}$ can be embedded in $R$ and also $R$ can be embedded in $U^{n}$. Then c.u.dim $(R)=$ c.u.dim $\left(U^{n}\right)$ and hence $R \cong U^{n}$, because $R$ is not uniform. Thus $U$ is a projective generator uniform right ideal of $R$. So $R$ is Morita equivalent to a domain. Now Lemma 4.11, Lemma 3.11 and Corollary 5.5 show that $R$ is simple artinian, a contradiction. So $R$ is not primitive, but every primitive factor ring is artinian (indeed all proper factor rings are artinian). Then since $R$ is a right V-ring, by [3], $R$ is regular and $\Sigma$-V-ring. Also every right ideal contains a non-zero uniform right ideal, hence minimal. So $R$ has non-zero essential $\operatorname{soc}(R)$. But $R$ is $\Sigma$-V-ring and by Corollary 3.19 , we have only finitely many nonisomorphic simple modules. Thus $\operatorname{soc}(R)$ is injective. This implies $R$ is semisimple, a contradiction. This completes the proof.

\section{Summary}

This paper defines couniserial dimension of a module that measures how far a module is from being uniform. The results proved in the paper demonstrate its importance for studding the structure of modules and rings and is a beginning of a larger project to study its impact. We close with some open questions:

(1) Does a module with arbitrary couniserial dimension possesses indecomposable dimension?

(2) Is there a theory for modules with both finite uniserial and couniserial dimensions that parallels to Krull-Schmidt-Remak-Azumaya theorem?

Acknowledgments The authors would like to thank the anonymous referee for the careful reading of this article and a lot of useful suggestions. This paper was written when the third author was visiting Ohio University, United States during May-August 2014. She wishes to express her deepest gratitude to 
Professor S. K. Jain for his kind guidance in her research project and Mrs. Parvesh Jain for the warm hospitality extended to her during her stay. She would also like to express her thanks to Professor E. Zelmanov for his kind invitation to visit the of University of Californian at San Diego and to give a talk.

Open Access This article is distributed under the terms of the Creative Commons Attribution License which permits any use, distribution, and reproduction in any medium, provided the original author(s) and the source are credited.

\section{References}

1. Anderson, F.W., Fuller, K.R.: Rings and Categories of Modules, 2nd ed. Graduate Texts in Mathematics, vol. 13. Springer, Berlin (1992)

2. Baccella, G.: On flat factor rings and fully right idempotent rings. Ann. Univ. Ferrara Sez. 26, 125-141 (1980)

3. Baccella, G.: Von Neumann regularity of V-rings with artinian primitive factor rings. Proc. Am. Math. Soc. 103(3), 747-749 (1988)

4. Cozzens, J., Faith, C.: Simple Noetherian Rings. Cambridge University Press, Cambridge (1975)

5. Dubrovin, N.I.: An example of a chain primitive ring with nilpotent elements. Mat. Sb. (N.S.) 120(162), no. 3, pp. 441-447 (1983) (Russian)

6. Ghorbani, A., Nazemian, Z.: On commutative rings with uniserial dimension. J. Algebra Appl. 14(1) (2015). doi:10.1142/S0219498815500085

7. Goldie, A.W.: Rings with Maximum Condition, Lecture Notes. Yale University, New Haven (1961)

8. Goodearl, K.R., Warfield, R.B., Jr.: An Introduction to Non commutative noetherian Rings, London Math. Soc., vol. 61. Cambridge University Press, Cambridge (2004)

9. Goodearl, K.R.: Ring Theory. Nonsingular Rings and Modules. Dekker, New York (1976)

10. Goodearl, K.R.: Surjective endomorphisms of finitely generated modules. Commun. Algebra. 15, 589-609 (1987)

11. Hart, R., Robson, J.C.: Simple rings and rings Morita equivalent to ore domains. Proc. Lond. Math. Soc. 21(3), 232-242 (1970)

12. Gordon, R., Small, L.W.: Piecewise domains. J. Algebra 23, 553-564 (1972)

13. Hirano, Y., Mogami, I.: Modules whose proper submodules are non-hopf kernels. Commun. Algebra. 15(8), 1549-1567 (1987)

14. Hirano, Y., Poon, E., Tsutsui, H.: A generalization of complete reducibility. Commun. Algebra. 40(6), 1901-1910 (2012)

15. Jain, S.K., Ashish Srivastava, K., Askar Tuganbaev, A.: Cyclic Modules and the Structure of Rings, Oxford Mathematical Monographs. Oxford University Press, Oxford (2012)

16. Lam, T.Y.: A crash course on stable range, cancellation, substitution, and exchange. J. Algebra Appl. 3, 301-343 (2004)

17. Lam, T.Y.: Lectures on Modules and Rings, Graduate Texts in Mathematics, vol. 189. Springer, New York (1999)

18. McConnell, J.C., Robson, J.C.: Noncommutative Noetherian Rings. Wiley, New York (1987)

19. Michler, G.O., Villamayor, O.E.: On rings whose simple modules are injective. J. Algebra 25, 185-201 (1973)

20. Nazemian, Z., Ghorbani, A., Behboodi, M.: Uniserial dimension of modules. J. Algebra 399, 894-903 (2014)

21. Stoll, R.R.: Set Theory and Logic. Dover Publication Inc, New York (1961)

22. Varadarajan, K.: Anti hopfian and anti coHopfian modules. AMS Contemp. Math. Ser. 456, 205-218 (2008)

23. Wisbauer, R.: Foundations of Module and Ring Theory. Gordon and Breach, Reading (1991) 